\title{
Luminometers for future linear collider experiments
}

\author{
Veta GHENESCU1 \\ Institute of Space Science, \\ 409 Atomistilor street, Bucharest-Magurele, RO-077125, ROMANIA \\ E-mail: ghenesculaspacescience.ro \\ Wolfgang LOHMANN \\ DESY, BTU Cottbus \& RWTH Aachen \\ Germany \\ E-mail: wlo@mail.cern.ch
}

\section{On behalf of the FCAL Collaboration}

In order to measure precisely and fast the luminosity in a future experiment at a linear collider like ILC or CLIC two compact and fine grained electromagnetic calorimeters are foreseen, LumiCal and BeamCal. LumiCal will provide a precise measurement of the integrated luminosity by counting Bhabha scattering events at small polar angles, while BeamCal is designed for bunch-by-bunch luminosity measurement and beam tuning when included in a fast feedback system using beam-strahlung. Both detectors will extend the detector coverage to small polar angles, potentially important for tagging high-energy electrons. The performance of a prototype of LumiCal, constructed as a sampling calorimeter with tungsten absorber plates of one $\mathrm{X}_{0}$ thickness and sensor planes with sub-mm thickness, was studied in an electron beam at DESY with momenta between one and $5 \mathrm{GeV}$. The shower position is measured with a precision of $440 \pm 20 \mu \mathrm{m}$, and the effective Molière radius is determined for electrons of $5 \mathrm{GeV}$ to be $8.1 \pm 0.3 \mathrm{~mm}$.

European Physical Society Conference on High Energy Physics - EPS-HEP2019 -

10-17 July, 2019

Ghent, Belgium

\section{${ }^{1}$ Speaker}




\section{Introduction}

Future $\mathrm{e}^{+} \mathrm{e}^{-}$linear colliders will allow precision measurements of interesting physics processes in a much cleaner environment than at a hadron collider. They will have the potential to study the spontaneous symmetry breaking with utmost precision and may manifest signatures of new physics from deviations of Standard model predictions or discovery of new particles with signatures hidden in the complex events of the LHC. Currently, two such colliders, distinguished by their acceleration concepts, are planned, the International Linear Collider (ILC) [1], based on superconducting cavities, and the Compact Linear Collider (CLIC) [2,3] with the two-beam accelerating concept and cavities operated at room temperature. Both colliders share two basic detector concepts, the Silicon Detector (SiD) [4] and the International Large Detector (ILD) [5]. The CLIC detector concept, referred to as the CLICdet, is adapted to the high-energy $\mathrm{e}+\mathrm{e}-$ collision environment at CLIC and optimised for particle flow reconstruction. The CLICdet concept was developed based on the lessons learnt from previous detector models for CLIC: CLIC_ILD and CLIC_SiD [6].

In all detector concepts, two compact and fine grained electromagnetic calorimeters at small polar angles are foreseen for the precise and fast measurements of the luminosity. The LumiCal, at larger polar angle, will count Bhabha events. Bhabha scattering is precisely calculated in QED, and knowing the cross section integrated over a certain polar angle range, the luminosity can be calculated. BeamCal, at lower polar angles, is hit by a large amount of low energy electrons or positrons, allowing at ILC a bunch-by-bunch estimate of the luminosity. BeamCal will also give input to a fastfeedback system to optimize the beam parameters. In addition, to support beam-tuning, a pair-monitor, positioned just in front of BeamCal, is foreseen. Both calorimeters will also improve the hermeticity of the detector, important e.g. to measure single high-energy electrons at small polar angles.

The FCAL collaboration developed a design for both calorimeters [7-9]. A sketch of the very forward region of the ILD detector is shown in Figure 1. LumiCal and BeamCal are designed as cylindrical electromagnetic sensor-tungsten sandwich calorimeters. Both consist of 30 tungsten absorber layers of $3.5 \mathrm{~mm}$ thickness, corresponding to one radiation length, interspersed with thin sensor layers. Each sensor layer is segmented in the radial and azimuthal directions, respectively, into pads. Front-end ASICs are positioned at the outer radius of the calorimeters. The BeamCal covers polar angles between 5 and $40 \mathrm{mrad}$ and the LumiCal between 31 and $77 \mathrm{mrad}$.

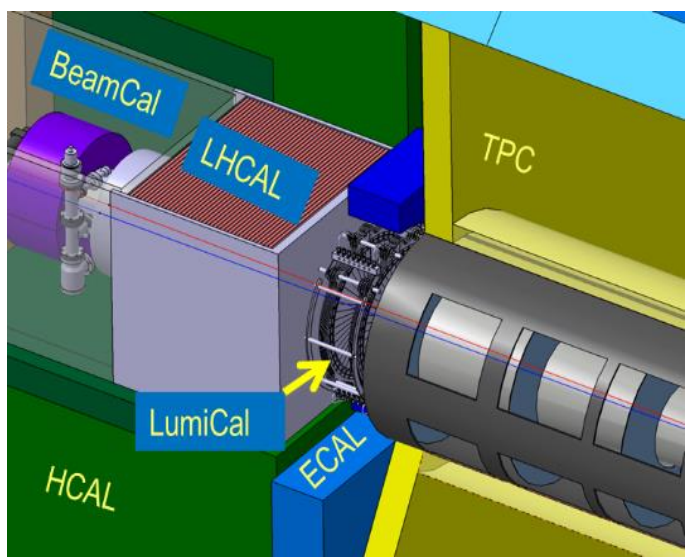

Figure 1. The very forward region of the ILD detector. The LumiCal, BeamCal, and LHCAL are carried by the support tube for the final focusing quadrupole and the beam-pipe. TPC denotes the central tracking chamber, ECAL the electromagnetic and HCAL the hadron calorimeter.

In LumiCal, silicon sensors will be used. BeamCal will be hit after each bunch crossing by beamstrahlung pairs, requiring radiation hard sensors. Currently the baseline option considers GaAs sensors. For both calorimeters, a small Molière radius is essential. In BeamCal, the depositions of 
single energetic electrons on top of the widely distributed depositions can be reconstructed with high efficiency. In LumiCal, a small Molière radius will reduce the polar angle range needed to define a fiducial volume with very good and constant performance.

This paper describes the construction of a LumiCal calorimeter prototype and the results from a beam-test carried out at the DESY II synchrotron using an electron beam with momenta between 1 and $5 \mathrm{GeV} / \mathrm{c}$.

\section{Beam-test set-up and results}

Data were collected in two test-beam campaigns in 2015 and 2016. A sketch of the beamline

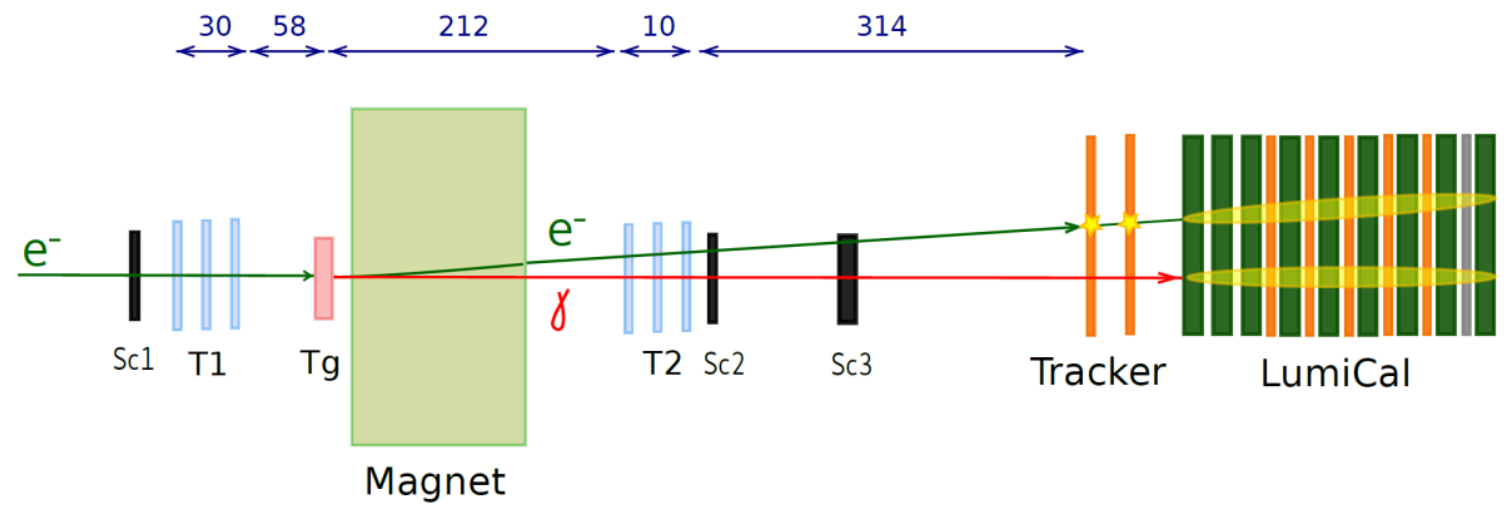

Figure 2. Sketch of the test-beam setup (not to scale).

instrumentation is shown in Figure 2. The electron beam passes through a $5 \times 5 \mathrm{~mm}^{2}$ square collimator that limits the beam spread along the test setup. The AIDA/EUDET beam telescope was placed upstream of the calorimeter. The telescope was split into two parts T1 and T2, each containing an arm with 3 layers of MIMOSA-26 pixel silicon detectors and 2 thin scintillator counters Sc1 and Sc2, for the trigger system. The telescope front arm was placed before the dipole magnet to record the incoming electrons. The rear arm was placed after the dipole magnet to record the electrons in the direction of the calorimeter, and to separate them from the photons generated in the copper target that was mounted just in front of the magnet.

The detector planes in the calorimeter consist of n-type silicon sensors with a thickness of $320 \mu \mathrm{m}$ shaped as a ring segment of 30 degrees subdivided into four sectors. Each sector is segmented in the radial direction in 64 pads of $1.8 \mathrm{~mm}$ pitch. The silicon sensor was glued with epoxy to a fanout made of flexible Kapton-copper foil. Ultrasonic wire bonding was used to connect conductive traces on the fanout to the sensor pads. A $70 \mu \mathrm{m}$ thick Kapton-copper foil glued on the back side of the sensor with a conductive glue supplied the high voltage. For good mechanical stability, the assembled sensor was embedded in a carbon fiber envelope. Figure 3 shows the structure of a detector plane. The fully assembled detector plane has a total thickness of $650 \mu \mathrm{m}$. 


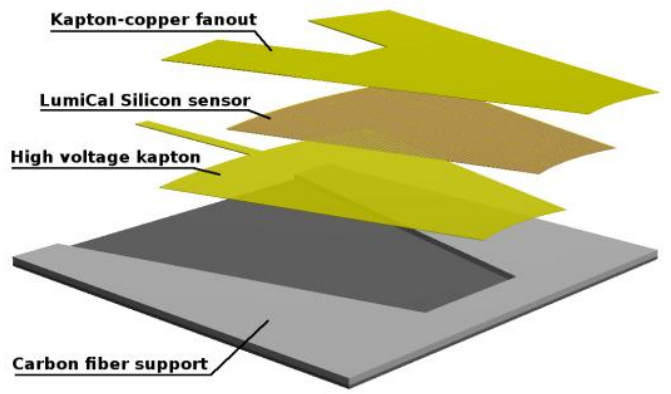

Figure 3. Structure of a detector plane.

Two detector planes placed in the lower part of Figure 4 are referred to as the "tracker" in Figure 2, used to reconstruct the electron tracks just in front of the calorimeter. The calorimeter prototype is seen in the upper part of Figure 4. Six sensor planes were installed in the $1 \mathrm{~mm}$ gap between the tungsten absorbers. APV25 front-end boards [10], used by the silicon strip detector of the CMS experiment, supplemented with charge dividers, were used for the readout as a temporary solution.

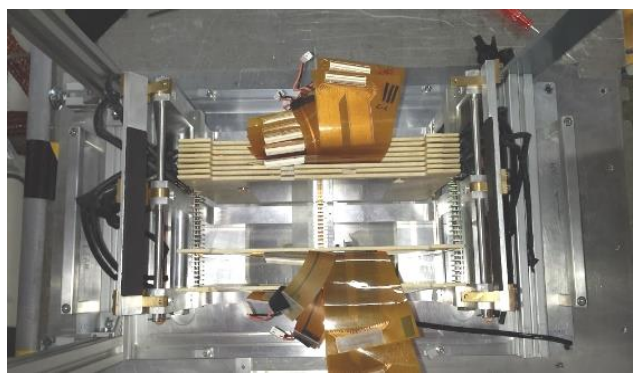

Figure 4. Top view of the tracker and LumiCal set-up.

More than seven million events were collected for different configurations of absorbers and readout planes, to measure the longitudinal and transversal shower development, the shower position and the effective Molière radius. The resolution of the shower position measurement was obtained from the distribution of the difference of the particle impact point, predicted by the tracker planes. The shower position was measured to be $440 \pm 20 \mu \mathrm{m}$. The measurement of the effective Molière radius [11] was carried out for electrons of 1,3 and $5 \mathrm{GeV}$. Figure 5 shows the transverse shower profile for beam energies of 1,3 and $5 \mathrm{GeV}$, as a function of the distance from the shower core. Data are nicely described by Monte Carlo simulations.

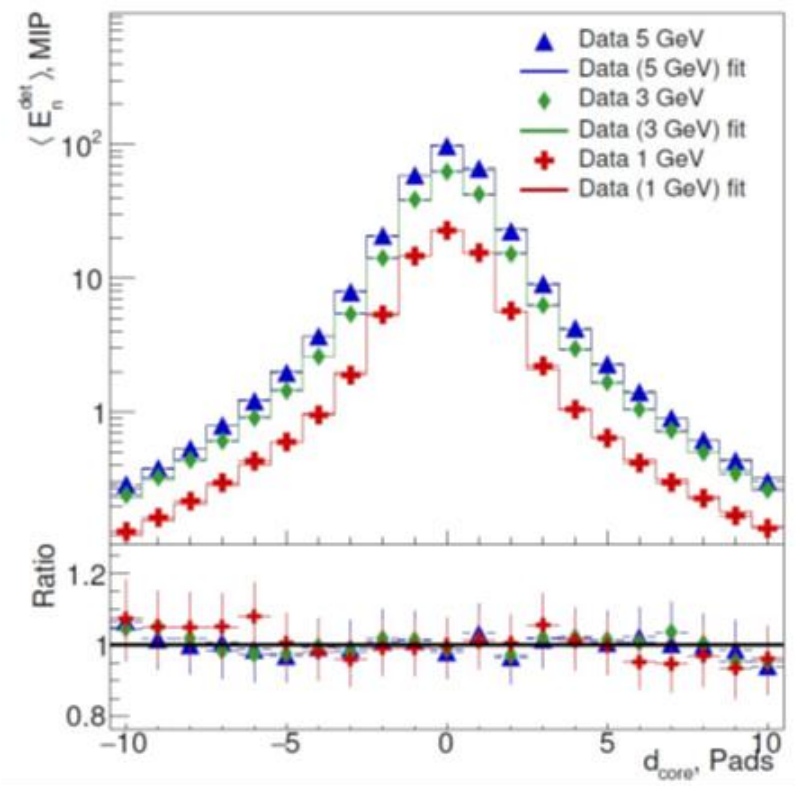

Figure 5. Average transverse shower profile as a function of $d_{\text {core }}$ in units of pads, for different beam energies. The lower portion of the figure displays the ratio between data and fit functions. 
The effective Molière radius is found to be $8.1 \pm 0.1 \mathrm{~mm}$ for $5 \mathrm{GeV}$ energy, a value in agreement with $8.4 \pm 0.1 \mathrm{~mm}$ [12] obtained through MC simulations. A slight dependence of the Molière radius on the electron energy is understood to be caused by the limited number of detector layers installed.

\section{Conclusions}

The design and performance of the LumiCal calorimeter prototype tested at the DESY-II Synchrotron with an electron beam with energies between 1 and $5 \mathrm{GeV}$ is presented. Thin sensor planes of $650 \mu \mathrm{m}$ thickness are positioned in the $1 \mathrm{~mm}$ gaps between tungsten absorber plates. The effective Molière radius was determined to be $8.1 \pm 0.1 \mathrm{~mm}$ being close to the technological limit. Currently dedicated readout ASICs in $130 \mathrm{~nm}$ technology are produced and prepared for the use in forthcoming test-beams. Finally, the performance of a fully equipped prototype will be studied.

\section{Acknowledgments}

The measurements leading to these results have been performed at the Test Beam Facility at DESY Hamburg (Germany), a member of the Helmholtz Association (HGF). This work was partially supported by the Romanian UEFISCDI agency under 18PCCDI 2018 project and MCI by the Nucleu programme, grant no. 16N/2019, and by the European Union Horizon 2020 Research and Innovation programme under Grant Agreement no.654168 (AIDA-2020). The measurements leading to these results have been performed at the Test Beam Facility at DESY Hamburg (Germany), a member of the Helmholtz Association (HGF).

\section{References}

[1] The International Linear Collider. Technical Design Report, Volume 2: Physics. 2013. arXiv:1306.6352

[2] Detector Technologies for CLIC, CERN Yellow Reports: Monographs, Vol. 1/2019, CERN-2019-001 (CERN, Geneva, 2019), http://dx.doi.org/10.23731/CYRM-2019-001

[3] The Compact Linear Collider (CLIC) - 2018 Summary Report, CERN Yellow Reports: Monographs, Vol. 2/2018, CERN-2018-005-M (CERN, Geneva, 2018), http://dx.doi.org/10.23731/CYRM-2018-002

[4] H. Aihara et al., SiD Letter of Intent, SLAC-R-989, 2009, arXiv: 0911.0006 [physics.ins-det].

[5] T. Abe et al., ILD Concept Group, The International Large Detector: Letter of Intent, DESY-2009-87, 2010, arXiv: 1006.3396 [hep-ex].

[6] L. Linssen et al., eds., CLIC Conceptual Design Report: Physics and Detectors at CLIC, CERN-2012-003, CERN, 2012, arXiv: 1202.5940 [physics.ins-det].

[7] H. Abramowicz et al., Instrumentation of the very forward region of a linear collider detector. IEEE Transactions on Nuclear Science, 51(6) (2004) 2983-2989.

[8] Forward Calorimetry Group (FCAL). R\&D for the ILC-Detector: Instrumentation of the Very Forward Region. http://www.desy.de/prc, 2006.

[9] H. Abramowicz et al., Performance of fully instrumented detector planes of the forward calorimeter of a Linear Collider detector, JINST 10 (2015) P05009.

[10] S. Martoiu, H. Muller, and J. Toledo, Front-end electronics for the Scalable Readout System of RD51, Proc. IEEE Nucl. Sci. Symp. Conf. Rec., Valencia, October 2011, pp 2036-2038 (2011), http://dx.doi.org/10.1109/NSSMIC.2011.6154414.

[11] H. Abramowicz et al., Measurement of shower development and its Moli'ere radius with a four-plane LumiCal test set-up. Eur.Phys. J. C 78 (2018) 135.

[12] H. Abramowicz et al., Performance and Molière radius measurements using a compact prototype of LumiCal in an electron test beam, Eur.Phys. J. C 79 (2019) 579. 